Full Paper

\title{
Dynamic Modelling of Nickel Complexation in Xylem Sap of Quercus ilex: A Voltammetric Study
}

\author{
Margarida Maria Correia dos Santos, ${ }^{\mathrm{a} *}$ Sheila Alves, ${ }^{\mathrm{a}}$ Maria de Lurdes Simões Gonçalves, ${ }^{\mathrm{a}}$ Cristina Nabais ${ }^{\mathrm{b}}$ \\ a Centro de Química Estrutural, Instituto Superior Técnico, Av. Rovisco Pais, 1049-001, Lisboa, Portugal \\ b Departamento de Botânica, Faculdade de Ciências e Tecnologia, Universidade de Coimbra, 3000 Coimbra, Portugal \\ *e-mail: mcsantos@alfa.ist.utl.pt
}

Received: October 21, 2005

Accepted: December 28, 2005

\begin{abstract}
Holm oak (Quercus ilex) is the dominant tree growing on serpentine soils of northeast Portugal, characterized by elevated soil concentrations of $\mathrm{Ni}$ and $\mathrm{Mg}$, combined with low Ca concentrations. Apparently $Q$. ilex does not suffer from excessive concentrations of $\mathrm{Ni}$ in the soil.

In this work we report a complexation study of nickel by the relevant ligands present in xylem sap: histidine, aspartic acid, oxalic and citric acids, at $0.10 \mathrm{M}$ ionic strength and $\mathrm{pH}$ 5.5. Single and mixed complexes were characterized. To validate the proposed complexation model, diluted solutions of $Q$. ilex xylem sap were titrated with nickel. All studies were done using square-wave voltammetry (SWV) at a hanging mercury drop electrode. Due to the dynamic nature of SWV, it is possible to obtain the conditional stability constants of the complexes formed but also to have knowledge on the kinetics of the interconversion of the species present.
\end{abstract}

Keywords: Nickel, Quercus ilex, Xylem sap, Voltammetry, Mixed complexes, Semidynamic complexes, Inert complexes

DOI: $10.1002 /$ elan.200503446

\section{Introduction}

Serpentine soils are known to be unfavorable to plant growth and productivity. Commonly they have high levels of trace metals like $\mathrm{Ni}, \mathrm{Cr}$ and $\mathrm{Co}$ and low $\mathrm{Ca} / \mathrm{Mg}$ ratios. In addition, serpentine soils have low concentrations of important nutrients like K and P. Elevated soil concentrations of $\mathrm{Ni}$ and $\mathrm{Mg}$, combined with low $\mathrm{Ca}$ concentrations are considered to be the main cause of serpentine soil toxicity [1]. However, Holm oak (Quercus ilex) the dominant tree growing on the serpentine soils of northeast Portugal does not suffer from excessive concentrations of $\mathrm{Ni}$ in the soil [2]. Nonsignificant differences in Ni concentration in leaves and xylem sap of $Q$. ilex from serpentine and nonserpentine soils are observed. Differences in Ni concentration are found in wood rings and bark of $Q$. ilex with trees growing in serpentine soils showing higher values when compared to trees of nonserpentine soils [3]. A better understanding of a possible mechanism of nickel transport, allocation and tolerance in $Q$. ilex trees surely involves knowledge of nickel binding by the relevant ligands present in xylem sap.

Xylem sap is mainly an aqueous solution where constituents like $\mathrm{Ca}, \mathrm{Mg}, \mathrm{K}$, nitrate, phosphates amino and carboxylic acids are found in millimolar concentrations ranges, while other elements like $\mathrm{Fe}, \mathrm{Zn}, \mathrm{Cu}$ and $\mathrm{Mn}$ may occur in micromolar concentration. The principal metal- binding ligands in plant xylem sap are small molecules like amino acids and carboxylic acids [4].

In xylem sap of $Q$. ilex, dominant carboxylic acids are malic, oxalic and citric with concentrations in the range 0.1 $1 \mathrm{mM}$. As to amino acids, aspartic acid concentration can reach values up to $100 \mu \mathrm{M}$ whereas histidine is less predominant and occurs in concentrations between 10 to $30 \mu \mathrm{M}$. No differences in xylem sap concentrations are observed in trees growing on serpentine and nonserpentine soils $[3,5]$. In this work we report a complexation study of nickel by the amino acids histidine (His), and aspartic acid (Asp) and the carboxylic acids oxalic (Ox) and citric (Cit) at $0.10 \mathrm{M} \mathrm{KNO}_{3}$ ionic strength and $\mathrm{pH}$ 5.5. These conditions were chosen since they correspond to average values of ionic strength and $\mathrm{pH}$ of $Q$. ilex xylem sap [3,5]. The total concentrations used, for both metal and ligands, were experimental average values found in xylem sap of $Q$. ilex grown on serpentine soils and collected monthly, during a period of one year. All values are shown in Table $1[3,5]$. The complexation was analyzed from solutions containing the individual ligands as well as from solutions containing a mixture of the above compounds that might be considered as a model solution of $Q$. ilex xylem sap. Also, the occurrence of mixed complexes was analyzed from experiments where two different ligands were used simultaneously. To validate the proposed complexation model, diluted solutions of $Q$. ilex xylem sap were titrated with nickel. All 
Table 1. Field concentrations of nickel and of low molecular weight organic compounds in the xylem sap of Quercus ilex grown on serpentine soils $[3,6]$.

\begin{tabular}{llll}
\hline Compound in xylem sap & Minimum concentration (M) & Maximum concentration (M) & Mean concentration (M) \\
\hline Nickel & $2.9 \times 10^{-6}$ & $1.3 \times 10^{-5}$ & $6.0 \times 10^{-6}$ \\
Aspartic acid & $2.9 \times 10^{-5}$ & $1.1 \times 10^{-4}$ & $6.5 \times 10^{-5}$ \\
Glutamic acid & $2.8 \times 10^{-5}$ & $9.5 \times 10^{-5}$ & $5.9 \times 10^{-5}$ \\
Asparagine & $1.4 \times 10^{-5}$ & $6.9 \times 10^{-5}$ & $4.5 \times 10^{-5}$ \\
Glutamine & $3.6 \times 10^{-6}$ & $9.9 \times 10^{-5}$ & $4.2 \times 10^{-5}$ \\
Histidine & $6.5 \times 10^{-6}$ & $3.3 \times 10^{-5}$ & $2.0 \times 10^{-5}$ \\
Arginine & $9.5 \times 10^{-6}$ & $6.3 \times 10^{-5}$ & $2.4 \times 10^{-5}$ \\
Leucine & $2.3 \times 10^{-6}$ & $3.9 \times 10^{-5}$ & $8.8 \times 10^{-6}$ \\
Lysine & $4.8 \times 10^{-6}$ & $1.5 \times 10^{-5}$ & $8.1 \times 10^{-6}$ \\
Tyrosine & $1.5 \times 10^{-6}$ & $7.6 \times 10^{-6}$ & $4.4 \times 10^{-6}$ \\
Valine & $2.5 \times 10^{-6}$ & $8.8 \times 10^{-6}$ & $5.8 \times 10^{-6}$ \\
Serine & $2.9 \times 10^{-6}$ & $1.3 \times 10^{-5}$ & $8.5 \times 10^{-6}$ \\
Threonine & $2.6 \times 10^{-6}$ & $1.4 \times 10^{-5}$ & $4.7 \times 10^{-6}$ \\
Alanine & $6.3 \times 10^{-6}$ & $8.3 \times 10^{-5}$ & $2.3 \times 10^{-5}$ \\
Citric acid & $5.0 \times 10^{-5}$ & $1.0 \times 10^{-3}$ & $5.0 \times 10^{-4}$ \\
Oxalic acid & $2.7 \times 10^{-4}$ & $1.6 \times 10^{-3}$ & $6.1 \times 10^{-4}$ \\
Malic acid & $2.9 \times 10^{-4}$ & $2.0 \times 10^{-3}$ & $1.1 \times 10^{-3}$ \\
\hline
\end{tabular}

complexation studies were done using square-wave voltammetry (SWV) at a hanging mercury drop electrode.

Although it is well known that biological effects of trace elements are greatly influenced by their chemical speciation, the bioavailability of metals in complex media is not only concerned with the equilibrium concentration of chemical species, but also with the dynamics of transformation among species and both effects must be taken into account $[6,7]$. Due to the dynamic nature of voltammetric methods, and in particular of SWV [8], it is possible to obtain information about the conditional stability constants of the complexes formed but also to have knowledge on the kinetics of the interconversion of the species present.

\section{Experimental}

\subsection{Chemicals and Solutions}

All solutions were prepared from analytical grade reagents using distilled and deionized water from a Millipore $\mathrm{Q}$ water purification system. Stock solutions of $0.5 \mathrm{M}$ nickel were prepared by dissolving nickel nitrate (BDH Chemicals) in deionized water and checked with an EDTA standard solution [9]. The supporting electrolyte was $0.10 \mathrm{M} \mathrm{KNO}_{3}$, adjusted to $\mathrm{pH} 5.5$ by the addition of either $\mathrm{KOH}$ or $\mathrm{HNO}_{3}$. Stock solutions of the amino acids $(0.10 \mathrm{M}$ histidine and $0.04 \mathrm{M}$ aspartic acid) and $0.10 \mathrm{M}$ carboxylic acids (citric and oxalic) were prepared by dissolving Merck reagents in the supporting electrolyte and were kept under refrigeration. Dilute solutions were prepared daily from the above stock solutions.

\subsection{Instruments}

Voltammetric measurements were performed using a potentiostat/galvanostat from ECO-Chimie, Autolab/ PGSTAT10 connected to the Methrom Stand 663 featuring a conventional three-electrode configuration: a Static Mercury Drop Electrode (SMDE) as the working electrode, an $\mathrm{Ag} / \mathrm{AgCl} / \mathrm{KCl}_{\text {(sat) }}$ as the reference electrode and a carbon rod auxiliary electrode.

Square Wave Voltammetry (SWV) was used in the complexation studies with a square-wave amplitude, $E_{\mathrm{sw}}$ of $25 \mathrm{mV}$, step height, $\Delta E, 5 \mathrm{mV}$, and different frequency, $f$, in the range $10-350 \mathrm{~Hz}$. In all experiments, the initial potential was $-0.8 \mathrm{~V}$ and the final potential was $-1.3 \mathrm{~V}$ vs. $\mathrm{Ag} /$ $\mathrm{AgCl}$.

Adsorption of carboxylic acids and xylem sap samples on mercury electrode surface was studied by alternatingcurrent polarography (AC). The measurements were performed in carboxylic acid solutions, xylem saps and xylem sap diluted solutions in $\mathrm{KNO}_{3}$ in the absence of nickel. An alternating potential of $10 \mathrm{mV}$ with a frequency of $50 \mathrm{~Hz}$ was superimposed on a DC ramp voltage that varied between 0.1 to $-1.5 \mathrm{~V}$ vs. $\mathrm{Ag} / \mathrm{AgCl}$. The alternating component of the current, $i_{\mathrm{ac}}$, was recorded in that potential range at a phase angle of $90^{\circ}$.

The $\mathrm{pH}$ measurements were carried out using an Orion $720 \mathrm{~A}$ digital $\mathrm{pH}$ meter coupled with an Orion $91-03 \mathrm{pH}$ electrode.

\subsection{Procedure}

\subsubsection{Xylem Sap Extraction and Composition}

Xylem sap sampling from $Q$. ilex growing in pots was done as described in $[3,5]$ using an optimized version of the vacuum extraction method of Bollard [10]. 
Total nickel concentration in xylem sap samples was determined by electrothermal atomic absorption.

High performance liquid chromatography was used in the determination of carboxylic [3] and amino acids [3,5].

\subsubsection{Complexation Studies}

In model solutions, voltammetric titrations were carried out in $0.10 \mathrm{M} \mathrm{KNO}_{3}$ and $\mathrm{pH} 5.5$ in different ways: a) to solutions with a constant total nickel concentration, $[\mathrm{M}]_{\mathrm{t}}$, successive volumes of ligand were added thus varying total ligand concentration, $[\mathrm{L}]_{\mathrm{t}}$, and the ratio of ligand to metal according to experimental field values; $b$ ) in solutions with constant ligand concentration (individual or mixtures of ligands), voltammetric measurements were performed in the presence of increasing amounts of nickel; c) mixed complexes formation was investigated in solutions containing two different ligands, by varying the concentration of one ligand while the concentration of the other as well as total nickel were kept constants.

In diluted solutions of xylem sap, voltammetric titrations were performed in $0.10 \mathrm{M} \mathrm{KNO}_{3}$ in the presence of increasing amounts of nickel. The proper dilution was determined after the AC polarography results.

After each ligand or metal addition, equilibrium attainment was checked recording the voltammetric signal at different time intervals. It was observed that in all situations superimposed voltammograms were obtained in less than 5 minutes. So, this was the equilibration time used.

$\mathrm{pH}$ was always measured for each experimental point upon metal or ligand addition and adjusted whenever necessary to 5.5 using $\mathrm{HNO}_{3}$ or $\mathrm{KOH}$.

Calibration of the voltammetric response to $\mathrm{Ni}$ was done in the absence of ligands at the same ionic strength and $\mathrm{pH}$ by plotting the SWV peak currents as a function of total nickel added.

All measurements were done in deaerated solutions with $\mathrm{U}$ type nitrogen and in a climatized room $\left(22 \pm 1^{\circ} \mathrm{C}\right)$.

\section{Results and Discussion}

\subsection{Simple Ligand Complexes}

\subsubsection{Voltammetric Behavior}

Since nickel(II) reduction at mercury is not a reversible process and the electron transfer rate constant can strongly vary with conditions, the voltammetric behavior was carefully analyzed from solutions containing just the supporting electrolyte (noncomplexing medium) and in the presence of each (or mixtures) of the four main organic constituents of $Q$. ilex xylem sap.

In all media, $\mathrm{Ni}(\mathrm{II})$ reduction is an irreversible process. This could be confirmed through the absence of backward current of the SW voltammograms and the cathodic shift of peak potentials, $E_{\mathrm{p}}$, with the increase of frequency [11]. Plots of $E_{\mathrm{p}}$ as a function of $\log f$ were linear, with slopes $=2.3 R T /$ $\alpha n F=32 \pm 2 \mathrm{mV}$ (where $\alpha$ is the transfer coefficient and other symbols have their usual meaning [8]) in the absence and presence of the ligands. Moreover, peak widths at half height, $W_{1 / 2}$, were the same both in noncomplexing and complexing media and equal to $105 \pm 5 \mathrm{mV}$.

However, nickel complexation by xylem sap components does affect the voltammetric behaviour, depending on the type of ligand and/or the time scale, $t_{\mathrm{m}}$.

In the case of the amino acids, and within the range of frequencies used, peak current due to $\mathrm{Ni}$ reduction decreases with the ligand addition. In all situations, peak potentials and half widths of SW voltammograms are not affected within the experimental error $(\Delta E / 2 \mathrm{mV})$. This can be seen in Figure 1a, where experimental SW voltammograms due to $\mathrm{Ni}$ reduction in the presence of increasing
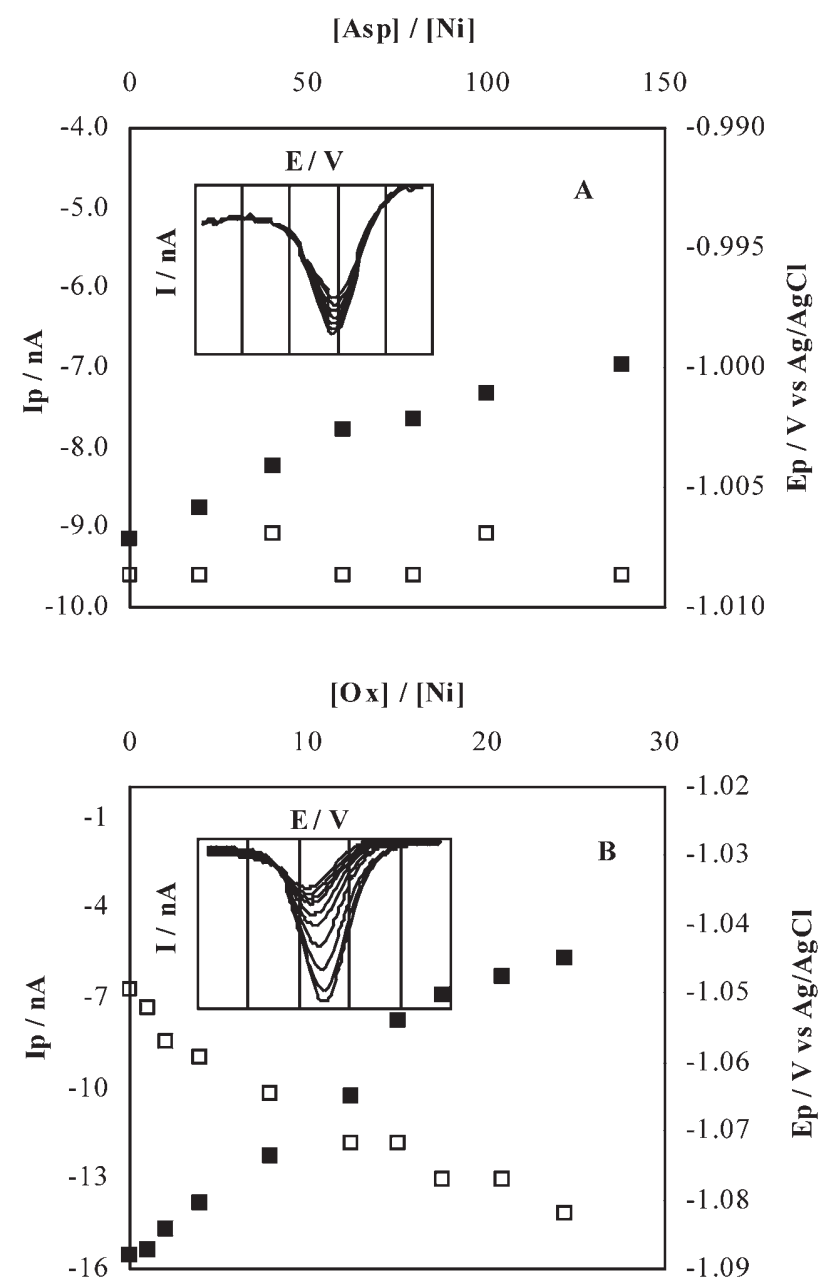

Fig. 1. Variation of nickel SW peak current (-) and peak potential $(\square)$ with the ratio $[\mathrm{L}]_{\mathrm{t}} /[\mathrm{M}]_{\mathrm{t}}$. Inset: SW voltammograms of nickel titration with: A) aspartic acid. $[\mathrm{M}]_{\mathrm{t}}=2.5 \times 10^{-6} \mathrm{M}$; $[\mathrm{L}]_{\mathrm{t}},=0 \mathrm{M}, 2.5 \times 10^{-5} \mathrm{M}, 5.0 \times 10^{-5} \mathrm{M}, 7.5 \times 10^{-5} \mathrm{M}, 1.5 \times 10^{-4} \mathrm{M}$, $2.5 \times 10^{-4} \mathrm{M}, 3.4 \times 10^{-4} \mathrm{M}$. B) Oxalic acid, $[\mathrm{M}]_{\mathrm{t}}=1.5 \times 10^{-5} \mathrm{M}$; $[\mathrm{L}]_{\mathrm{t}}=0 \mathrm{M}, 7.6 \times 10^{-6} \mathrm{M}, 3.0 \times 10^{-5} \mathrm{M}, 6.0 \times 10^{-5} \mathrm{M}, 1.2 \times 10^{-4} \mathrm{M}$, $1.5 \times 10^{-4} \mathrm{M}, \quad 2.3 \times 10^{-4} \mathrm{M}, \quad 2.6 \times 10^{-4} \mathrm{M}, \quad 3.2 \times 10^{-4} \mathrm{M}, \quad 3.7 \times$ $10^{-4} \mathrm{M}$. Voltammetric conditions: $E_{\mathrm{i}}=-800 \mathrm{mV} ; \quad E_{\mathrm{f}}=$ $-1300 \mathrm{mV} ; E_{\mathrm{SW}}=25 \mathrm{mV} ; \Delta E=5 \mathrm{mV} ;$ frequency $=10 \mathrm{~Hz} ; \quad T=$ $22 \pm 1{ }^{\circ} \mathrm{C}$; electrolyte: $0.10 \mathrm{M} \mathrm{KNO}_{3}$; $\mathrm{pH}$ 5.5. 
amounts of aspartic acid are also presented in the inset. So, the voltammetric behaviour found for $\mathrm{Ni}$ in the presence of these amino acids is indicative of an inert behavior. The metal complexes formed behave as nonlabile in the voltammetric conditions used, i.e., they are electrochemically not available to the mercury electrode [12].

With complex systems like the present ones, one might expect the occurrence of ligand-induced adsorption phenomena that might seriously affect the voltammetric signal. Adsorption studies previously reported [13] showed that there is no significant adsorption on mercury of aspartic acid and histidine over the entire range of potential for the concentration levels and $\mathrm{pH}$ used in this work. Additionally, evidence was now obtained through the variation of $\mathrm{Ni}$ (II) square-wave peak current, $i_{\mathrm{p}}$, with the increase of frequency in the absence and presence of amino acids. In all media, good linear relationships (correlation coefficient $r>0.99$ ) with null intercepts within the experimental errors (confidence level $95 \%$ ) were obtained for $i_{\mathrm{p}}$ with the square root of $f$. This behavior means that $\mathrm{Ni}$ (II) reduction is not affected by adsorption [14] and the voltammetric signal intensity is directly related to free metal ion concentration in solution [12].

In the presence of either oxalic or citric acids, a different behavior was found depending on the frequency and/or the concentration. For the highest frequencies, and for the lowest concentrations, the voltammetric signal due to $\mathrm{Ni}$ reduction behaved as described above. However, for $f=$ $10 \mathrm{~Hz}$, upon carboxylic acid additions, peak current of SW voltammograms decreased and simultaneous there was a shift of peak potentials towards negative values. This can be seen in Figure $1 \mathrm{~b}$, that also shows, in the inset, the SW voltammograms due to $\mathrm{Ni}$ reduction obtained in the presence of increasing amounts of oxalic acid at $f=10 \mathrm{~Hz}$. In these circumstances, the complexes behaved as non-inert [12]. A similar behavior was previously reported in a polarographic study of $\mathrm{Ni}$ reduction in the presence of oxalate ion [15].

The differences described are not surprising since the dynamic behavior of the complexes formed depends not only on the kinetics of the dissociation process but also on the time scale, $t_{\mathrm{m}}$, of the technique [7].

For a general complexation reaction between a metal $\mathrm{M}$ and a ligand $\mathrm{L}$ described by:

$$
\mathrm{M}+\mathrm{L} \underset{k_{d}}{\stackrel{k_{a}}{\rightleftarrows}} \mathrm{ML}
$$

The conditional formation constant $K$ of the complex ML is given by:

$$
K=[\mathrm{ML}] /[\mathrm{M}] \cdot[\mathrm{L}]=k_{\mathrm{a}} / k_{\mathrm{d}}
$$

where $k_{\mathrm{a}}$ and $k_{\mathrm{d}}$ are the formation and dissociation rate constants, respectively. In the presence of an excess of ligand one has $k_{\mathrm{a}}{ }^{\prime}=k_{\mathrm{a}}[\mathrm{L}]$, the pseudo first order formation rate constant.
If $t_{\mathrm{m}}$ is the time scale of the technique $\left(t_{\mathrm{m}}=(1 /(2 f))\right.$, different voltammetric behaviors will be found depending on the products $k_{\mathrm{d}} t_{\mathrm{m}}$ and $k_{\mathrm{a}}{ }^{\prime} t_{\mathrm{m}}$ [7]:

$k_{\mathrm{d}} t_{\mathrm{m}}, k_{\mathrm{a}}{ }^{\prime} t_{\mathrm{m}} \ll 1 \rightarrow$ inert/static complex

$k_{\mathrm{d}} t_{\mathrm{m}}, k_{\mathrm{a}}^{\prime} t_{\mathrm{m}} \gg 1 \rightarrow$ dynamic complex

$k_{\mathrm{d}} t_{\mathrm{m}}, k_{\mathrm{a}}{ }^{\prime} t_{\mathrm{m}} \cong 1 \rightarrow$ semidynamic complex

For small, not strongly binding ligands, it has been shown that dehydration of $\mathrm{M}\left(\mathrm{H}_{2} \mathrm{O}\right)_{\mathrm{q}}$ subsequent to the formation of an outer sphere complex is often the rate limiting step in the formation of the metal complex ML which is quite well known as the Eigen mechanism [16]. Under these conditions the formation rate constant can be estimated from the product of the water loss rate constant, $k_{-\infty}$ and of the outer sphere stability constant, $K_{\mathrm{OS}}$ :

$k_{\mathrm{a}}=K_{\mathrm{OS}} k_{-\omega}$

For $\mathrm{Ni}, k_{-\omega}=3 \times 10^{4} \mathrm{~s}^{-1}$ [17]. As to $K_{\mathrm{OS}}$, although for these ligands there is a distribution of charges over the ligand molecule, it can be estimated, as an approximation, from a simple electrostatic computation on the basis of point charges. Taking into account the charges of the reacting species, at the medium ionic strength and $\mathrm{pH}$, one has $K_{\mathrm{OS}}$ $($ Asp $)=2.1 \mathrm{~s}^{-1}, K_{\mathrm{OS}}(\mathrm{His})=5.0 \times 10^{-2} \mathrm{~s}^{-1}, K_{\mathrm{OS}}(\mathrm{Ox})=14 \mathrm{~s}^{-1}$ and $K_{\mathrm{OS}}(\mathrm{Cit})=14 \mathrm{~s}^{-1}$ [18]. Values for the dissociation rate constant can then be estimated from the thermodynamic equilibrium constant using calculated values of $k_{\mathrm{a}}$ and Equation 1.

Table 2 shows the values calculated for the products $k_{\mathrm{d}} t_{\mathrm{m}}$ and $k_{\mathrm{a}}{ }^{\prime} t_{\mathrm{m}}$, at $f=10$ and $f=100 \mathrm{~Hz}$ and maximum and minimum field concentrations of the ligands found in $Q$. ilex xylem sap. As to the formation constants, the values used in the calculations were those valid for the ionic strength and $\mathrm{pH}$ of xylem sap of $Q$. ilex and determined in this work (see below).

Analysis of the values shows that for the time scale used, of the order of ms, and for the range of the main amino acid and carboxylic acid concentrations present in $Q$ ilex xylem sap, Ni complexes with amino acids should behave as inert while those with oxalic and citric acids would behave in the boundary as semidynamic complexes. This is in very good agreement with the behavior found experimentally in the voltammetric runs.

\subsubsection{Calculation of the Conditional Stability Constants}

For inert/static complexes (Ni complexes with the amino acids), since the voltammetric intensity signal is directly related to free metal ion concentration in solution [12], conditional stability constants are determined directly from the peak current, $i_{\mathrm{p}}$, due to nickel reduction and using the mass balances equations for the metal ion and the ligand.

In the case of semidynamic complexes (Ni complexes with the carboxylic acids at $f=10 \mathrm{~Hz}$ ), conditional stability 
Table 2. $k_{\mathrm{a}}^{\prime} t$ and $k_{\mathrm{d}} t$ values for ML complexes of nickel in xylem sap conditions ([ $\left.\mathrm{KNO}_{3}\right]=0.10 \mathrm{M}, \mathrm{pH} 5.5$ and limit concentrations of ligand).

\begin{tabular}{|c|c|c|c|c|c|}
\hline Ligand (L) & [Ligand] (M) & $\begin{array}{l}k_{\mathrm{a}}^{\prime} t \\
10 \mathrm{~Hz}\end{array}$ & $100 \mathrm{~Hz}$ & $\begin{array}{l}k_{\mathrm{d}} t \\
10 \mathrm{~Hz}\end{array}$ & $100 \mathrm{~Hz}$ \\
\hline Aspartic acid & $\begin{array}{l}2.9 \times 10^{-5} \\
1.1 \times 10^{-4}\end{array}$ & $\begin{array}{l}9.1 \times 10^{-2} \\
3.4 \times 10^{-1}\end{array}$ & $\begin{array}{l}9.1 \times 10^{-3} \\
3.4 \times 10^{-2}\end{array}$ & $2.2 \times 10^{-4}$ & $2.2 \times 10^{-5}$ \\
\hline Histidine & $\begin{array}{l}6.5 \times 10^{-6} \\
3.3 \times 10^{-5}\end{array}$ & $\begin{array}{l}2.4 \times 10^{-3} \\
4.7 \times 10^{-3}\end{array}$ & $\begin{array}{l}2.4 \times 10^{-4} \\
4.7 \times 10^{-4}\end{array}$ & $1.5 \times 10^{-7}$ & $1.5 \times 10^{-8}$ \\
\hline Citric acid & $\begin{array}{l}5.0 \times 10^{-5} \\
1.0 \times 10^{-3}\end{array}$ & $\begin{array}{l}1.0 \\
20\end{array}$ & $\begin{array}{l}1.0 \times 10^{-1} \\
2.0\end{array}$ & $8.1 \times 10^{-2}$ & $8.1 \times 10^{-3}$ \\
\hline Oxalic acid & $\begin{array}{l}2.7 \times 10^{-4} \\
1.6 \times 10^{-3}\end{array}$ & $\begin{array}{l}5.0 \\
32\end{array}$ & $\begin{array}{l}5.5 \times 10^{-1} \\
3.0\end{array}$ & $8.8 \times 10^{-1}$ & $8.8 \times 10^{-2}$ \\
\hline
\end{tabular}

constants $K_{\mathrm{i}}$ are determined from the potential shift $\Delta E_{\mathrm{p}}$ and peak currents values according to Equation 6 [19].

$$
\begin{aligned}
\Delta E_{p} & =\left(E_{p}\right)_{c}-\left(E_{p}\right)_{s} \\
& =-\frac{R T}{\alpha n F} \ln \left(\sum_{i=0}^{j} K_{i}[L]^{i}\right)-\frac{R T}{\alpha n F} \ln \left(\frac{i_{k}}{i_{d}}\right)
\end{aligned}
$$

where $\left(E_{\mathrm{p}}\right)_{\mathrm{c}}$ and $\left(E_{\mathrm{p}}\right)_{\mathrm{s}}$ are the peak potentials due to $\mathrm{Ni}$ reduction in the presence and absence of ligand, respectively, and $i_{\mathrm{k}}$ and $i_{\mathrm{d}}$ are the corresponding peak currents. The above equation is valid for an excess of ligand, and since in this case the diffusion coefficient of both aquo-ion and complex species are similar, i.e., $D_{\mathrm{M}} \cong D_{\mathrm{ML}}$, it should be $[\mathrm{L}] /$ $[\mathrm{ML}]=10$, in order to have an error in $\Delta E_{\mathrm{p}}$ not higher then $5 \mathrm{mV}$ [19].

The conditional formation constants determined for the simple-ligand complexes are shown in Table 3 where values available in the literature are also presented for comparison. The good agreement between them is a good check of the results accuracy determined by SWV and it is important to validate the voltammetric data interpretation. If the shift in peak potential, which sometimes is overlooked in literature, had not been taken into account in the calculations, and so the complexes with the carboxylic acids considered as inert, the conditional formation constants would be affected by a systematic error. Lower values, of the order of 0.3 units, would be obtained for $\log K$.

Table 3. Conditional stability constants $(\log K)$ of nickel complexes determined by SWV. Voltammetric conditions: $E_{\mathrm{i}}=$ $-800 \mathrm{mV} ; E_{\mathrm{f}}=-1300 \mathrm{mV} ; E_{\mathrm{SW}}=25 \mathrm{mV} ; \Delta E=5 \mathrm{mV} ; 10 \mathrm{~Hz} \leq$ Frequency $\leq 100 \mathrm{~Hz}$; Electrolyte: $0.10 \mathrm{M} \mathrm{KNO} 3, \mathrm{pH}=5.5 ; T=$ $22 \pm 1{ }^{\circ} \mathrm{C}$.

\begin{tabular}{lllll}
\hline Ligand & $\log K_{1}$ & $\log K_{2}$ & $\log K_{1}[20]$ & $\log K_{2}[20]$ \\
\hline Asp & $7.03 \pm 0.04$ & - & 7.16 & 12.40 \\
His & $8.63 \pm 0.03$ & - & $8.67 \pm 0.02$ & $15.54 \pm 0.04$ \\
Cit & $\left.5.58 \pm 0.03^{\text {a }}\right)$ & $10.00 \pm 0.07^{\text {b) }}$ & $5.25 \pm 0.2$ & 8.11 \\
Ox & $4.49 \pm 0.03$ & $7.37 \pm 0.05$ & $4.26^{[15]}$ & $7.54^{[15]}$ \\
\hline
\end{tabular}

$\log K_{1}($ ap $)=\log \left(K_{1}+K_{\mathrm{MHL}}\left[\mathrm{H}^{+}\right]\right)$

$\log K_{2}(\mathrm{ap})=\log \left(K_{2}+K_{\mathrm{M}(\mathrm{HL}) 2}\left[\mathrm{H}^{+}\right]^{2}\right)$
For $\mathrm{Ni}+\mathrm{Asp}$ and $\mathrm{Ni}+$ His systems, the voltammetric results could be explained by the formation of $1: 1 \mathrm{com}-$ plexes. In the case of $\mathrm{Ni}+\mathrm{Ox}$ system, the formation of $1: 2$ complexes with a $K_{2} \approx 10^{7.37}$ was also detected. Due to $\mathrm{p} K_{\mathrm{a}}$ values of citric acid, $\mathrm{p} K_{\mathrm{a} 1}=2.87, \mathrm{p} K_{\mathrm{a} 2}=4.35$, and $\mathrm{p} K_{\mathrm{a} 3}=5.69$ [20], citrate is mainly present as $\mathrm{HL}^{2-}$ at $\mathrm{pH} 5.5$, which can bind to $\mathrm{Ni}$ forming $\mathrm{NiHL}$ as well as NiL. In the same way 1:2 complexes can occur.

\subsection{Mixed Ligand Complexes}

In samples where different ligands are present mixed-ligand complex formation is a general and common phenomenon. Actually, mixed ligands should be preferred over simple complexes whenever the concentrations of the ligands involved are such that the products of simple complexes conditional formation constants and ligands concentrations, raise to the appropriate power, are approximately equal [21].

Complex biological fluids like xylem sap are mixed ligand environments, so mixed-ligand complexes are important models for understanding metal reactivity in those systems.

The formation of mixed complexes was investigated in solutions containing His $+\mathrm{Cit}$, Asp + Ox and Ox + Cit, by varying the concentration of one ligand while the concentration of the other as well as the metal were kept constant. Those combinations were chosen taking into account statistic considerations, as well as Ni coordination chemistry and ligands characteristics [22, 23]. Nickel forms hexacoordinated complexes with ligands having nitrogen and/or oxygen atoms as donors, and mixed complexes with bidentate or tridentate ligands have been identified and studied by techniques such as potentiometry and spectrophotometry [24-30]. Voltammetric techniques, like polarography and differential pulse polarography, have also been used in the study of mixed complexes of other metals such as $\mathrm{Cd}$ and $\mathrm{Pb}$. In all situations complexes behaved as labile and the stability constants were evaluated using a generalization of DeFord and Hume's treatment for the study of simple complexes [31-35].

Figure 2a inset shows SW voltammograms at $f=100 \mathrm{~Hz}$, due to $\mathrm{Ni}$ reduction from solutions containing this metal ion and oxalic acid in a constant concentration while increasing 

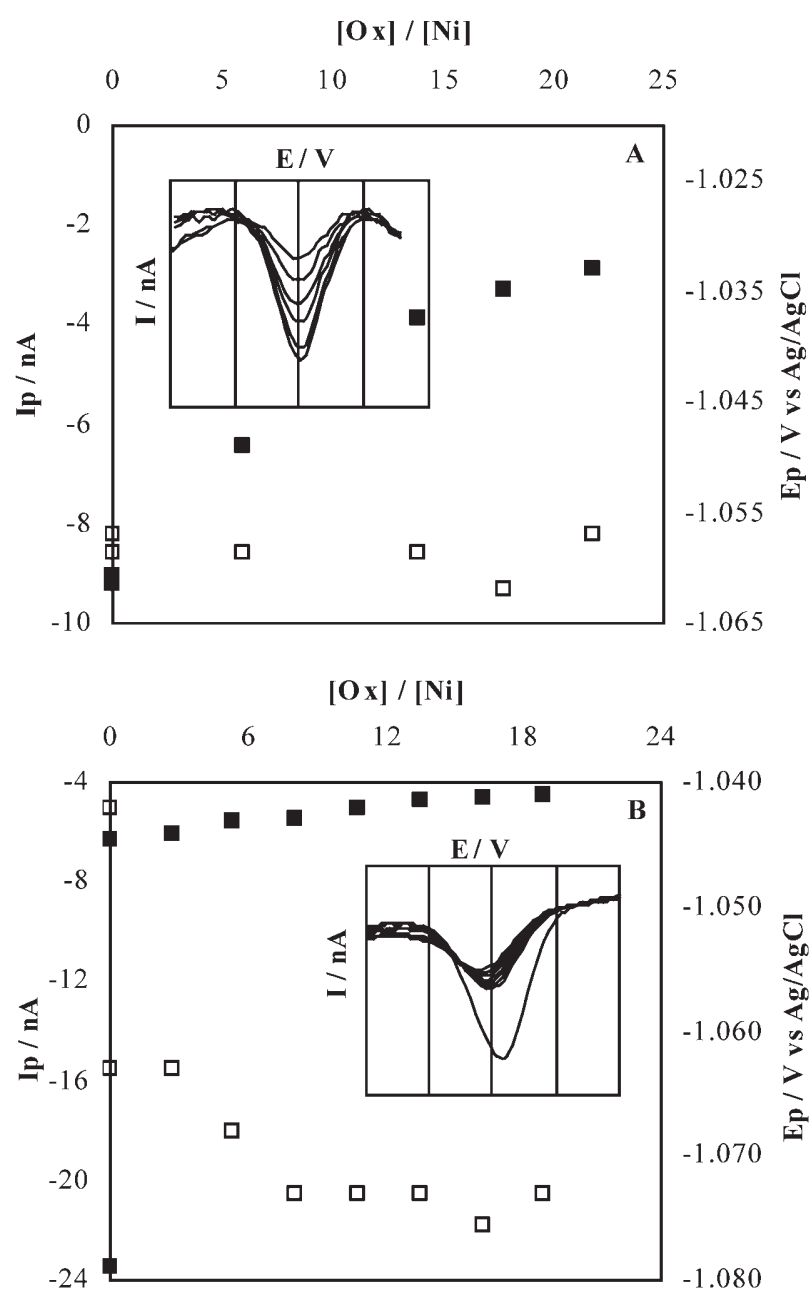

Fig. 2. Variation of nickel SW peak current (๘) and peak potential $(\square)$ with the ratio $[\mathrm{L}]_{\mathrm{t}} /[\mathrm{M}]_{\mathrm{t}}$. Inset: SW voltammograms of: A) nickel and aspartic acid (L) titration with oxalic acid (Y). $[\mathrm{M}]_{\mathrm{t}}=5.0 \times 10^{-6} \mathrm{M} ;[\mathrm{Asp}]_{\mathrm{t}}=0,6.5 \times 10^{-5} \mathrm{M} ;[\mathrm{Ox}]_{\mathrm{t}}=0 \mathrm{M}, 3.0 \times$ $10^{-5} \mathrm{M}, 6.9 \times 10^{-5} \mathrm{M}, 8.9 \times 10^{-5} \mathrm{M}, 1.1 \times 10^{-4} \mathrm{M}$. B) Nickel and citric acid $(\mathrm{L})$ titration with oxalic acid $(\mathrm{Y}) .[\mathrm{M}]_{\mathrm{t}}=1.0 \times 10^{-5} \mathrm{M}$; $[\mathrm{Cit}]_{\mathrm{t}}=0 \mathrm{M}, 5.0 \times 10^{-5} \mathrm{M},[\mathrm{Ox}]_{\mathrm{t}}=0 \mathrm{M}, 2.7 \times 10^{-5} \mathrm{M}, 5.4 \times 10^{-5} \mathrm{M}$, $8.1 \times 10^{-5} \mathrm{M}, \quad 1.1 \times 10^{-4} \mathrm{M}, \quad 1.3 \times 10^{-4} \mathrm{M}, \quad 1.6 \times 10^{-4} \mathrm{M}, \quad 1.9 \times$ $10^{-4} \mathrm{M}$. Voltammetric conditions: $E_{\mathrm{i}}=-800 \mathrm{mV} ; \quad E_{\mathrm{f}}=$ $-1300 \mathrm{mV} ; E_{\mathrm{Sw}}=25 \mathrm{mV} ; \Delta E=5 \mathrm{mV} ;$ frequency $=10 \mathrm{~Hz} ; T=$ $22 \pm 1{ }^{\circ} \mathrm{C}$; electrolyte: $0.10 \mathrm{M} \mathrm{KNO}_{3} ; \mathrm{pH}$ 5.5.

the concentration of aspartic acid. In the same figure, variation of both peaks potential and current with increasing amounts of the amino acid can be seen, i.e., peak potentials remain constant and equal to the $E_{\mathrm{p}}$ in the absence of ligand, while peak currents decrease. The same behavior was observed in solutions where aspartic acid concentration was kept constant while varying the carboxylic acid. The same happened for $\mathrm{Ni}+\mathrm{His}+\mathrm{Cit}$ system. So, in these situations the complexes behaved as inert, as happens in general when at least one of the ligands forms inert complexes [36], and then the peak current due to nickel reduction is directly related to the free metal ion concentration in solution.
For $\mathrm{Ni}+\mathrm{Ox}+\mathrm{Cit}$ system, the complexes behaved as semidynamic (Fig. 2b), i.e., upon ligand addition both peak current and potential vary in a similar way to that observed in solutions containing just one carboxylic acid (see Fig. 1b), i.e., peak current decreases while peak potential shift cathodically.

Determination of free metal ion concentration in solution had to be done through peak potential differences, $\Delta E_{\mathrm{p}}$, and currents. In order to calculate free nickel concentration, and since $\sum_{i=0}^{j} K_{i}[\mathrm{~L}]^{i}=[\mathrm{M}]_{t} /[\mathrm{M}]$, Equation 6 can be rewritten as:

$\Delta E_{\mathrm{p}}=(R T / \alpha n F) \ln \left([\mathrm{M}]_{\mathrm{t}} /[\mathrm{M}]\right)-(R T / \alpha n F) \ln \left(i_{\mathrm{k}} / i_{\mathrm{d}}\right)$

Assuming the formation of both mono and mixed complexes of the type MLY, considering the mass balances for $\mathrm{Ni}$ and ligands and working in conditions where $1: 2$ species are negligible, an overall constant can be defined as

$K^{\prime}=([\mathrm{ML}]+[\mathrm{MY}]+[\mathrm{MLY}]) /([\mathrm{M}][\mathrm{L}][\mathrm{Y}])=\left([\mathrm{M}]_{\mathrm{t}}-[\mathrm{M}]\right) /$ $([\mathrm{M}][\mathrm{L}][\mathrm{Y}])$

With the knowledge of $K_{\mathrm{ML}}$ and $K_{\mathrm{MY}}$, and in conditions where the complexes concentration formed with the ligand kept constant are negligible compared to free and protonated ligand, $K_{\mathrm{MLY}}$ can be easily computed from the intercept obtained by extrapolation of a plot of $K^{\prime}$ vs. 1/[Y] (while [L] is constant) or $K^{\prime}$ vs. $1 /[\mathrm{L}]$ (while [Y] is constant). These should be straight lines with a slope equal to the complex conditional formation constant with the ligand kept constant. Both situations are illustrated in Figure 3 where plots of $K^{\prime}$ against $1 /$ [ligand] are shown from the data obtained in the voltammetric titration of Ni solutions with a) aspartic acid at a constant oxalic acid concentration and with b) oxalic acid at a constant aspartic acid concentration. Similar representations were obtained for the other systems. In all situations, straight lines have been obtained with a correlation coefficient $r>0.99$ (confidence level 95\%). The good fit of the experimental data to Equation 8 confirms the assumptions made.

The values determined for the conditional formation constants are presented in Table 4. Since there are no experimental values in the literature, the comparison was made with the conditional formation constants of mixedcomplexes estimated on a statistical basis according to Equation 9 [23].

$\log K_{\mathrm{i}}=(l / L) \log K_{\mathrm{L}}+(y / Y) \log K_{\mathrm{Y}}+\log S$

Where $K_{\mathrm{i}}$ is the mixed species conditional stability constant; $l$ and $y$ are the number of ligands (L, Y) in the mixed complex; $L$ and $Y$ represent the number of ligands in corresponding simple complexes having the same metal to ligand ratio. $S$ is a statistical factor given by $n ! /(l ! y !)$, where $n$ is the total number of ligands in the complex species. As observed in other situations, it is interesting to note that the difference between experimental and calculated values of conditional stability constants is positive, probably due to ligand-ligand interactions [23]. 

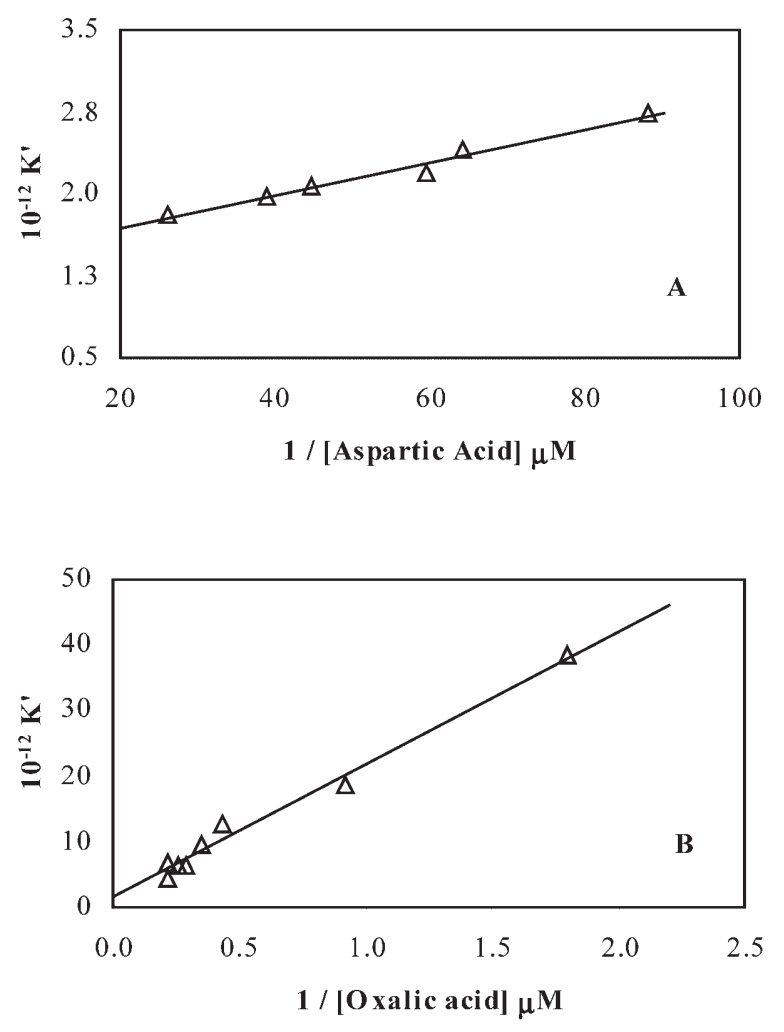

Fig. 3. Plots of $K^{\prime}$ against $1 /$ [Ligand] constructed from data obtained in voltammetric titration of $5.0 \times 10^{-6} \mathrm{M}$ nickel solutions with: A) constant oxalic acid concentration $\left(1.0 \times 10^{-5} \mathrm{M}\right), y=$ $(1.0 \pm 0.2) \times 10^{12}+(2.2 \pm 0.4) \times 10^{4} x, r=0.99$. B $)$ Constant aspartic acid concentration $\left(1.1 \times 10^{-4}\right) \mathrm{M}, y=(1.4 \pm 0.7) \times 10^{12}+(2.0 \pm$ $0.1) \times 10^{7} x, r=0.99$.

\subsection{Titration of a Model solution of Xylem Sap of $Q$. ilex with $\mathrm{Ni}$}

A model solution of $Q$. ilex xylem sap containing average concentrations of histidine, citric, oxalic and aspartic acids (as shown in Table 1), well buffered to $\mathrm{pH} 5.5$ due to the presence of citric acid, was titrated with $\mathrm{Ni}$. Increasing total nickel concentration there was an increase in peak current being the values, however, lower than the corresponding ones for the calibration plot. As to the peak potentials of SW voltammograms, a cathodic shift was observed which tends to decrease with the increase of nickel in solution. In order to calculate free nickel concentration, Equation 7 has to be used in the form:

$$
\Delta E_{\mathrm{p}}=(R T / \alpha n F) \ln \left([\mathrm{M}]_{\mathrm{t}}^{\mathrm{lab}} /[\mathrm{M}]\right)-(R T / \alpha n F) \ln \left(i_{\mathrm{k}} / i_{\mathrm{d}}\right)
$$

Where $i_{\mathrm{d}}$ is the current expected if the process is solely diffusion controlled and $[\mathrm{M}]_{\mathrm{t}}^{\text {lab }}$ is given by Equation 11:

$$
\begin{aligned}
& {[\mathrm{M}]_{\mathrm{t}}^{\mathrm{lab}}=[\mathrm{M}]+[\mathrm{MOx}]+\left[\mathrm{MOx}_{2}\right]+[\mathrm{MCit}]+\left[\mathrm{MCit}_{2}\right]+} \\
& {[\mathrm{MOxCit}]}
\end{aligned}
$$

Values of $[\mathrm{M}]_{\mathrm{t}}^{\text {lab }}$ are not directly known since nickel also forms complexes with the amino acids that are inert being the total mass balance for the metal given by Equation 12:

$$
\begin{aligned}
& {[\mathrm{M}]_{\mathrm{t}}=[\mathrm{M}]_{\mathrm{t}}^{\text {lab }}+[\mathrm{MAsp}]+[\text { MHis }]+[\text { MAspOx }]+} \\
& {[\text { MHisCit }]}
\end{aligned}
$$

However $i_{\mathrm{d}}$ is proportional to $[\mathrm{M}]_{\mathrm{t}}^{\text {lab }}$ according to:

$i_{\mathrm{d}}=s[\mathrm{M}]_{\mathrm{t}}^{\mathrm{lab}}$

where $s$ is the calibration plot slope since $D_{\mathrm{M}} \cong D_{\mathrm{ML}}$. Combining Equations 10 and 13 one obtains Equation 14:

$\Delta E_{\mathrm{p}}=(R T / \alpha n F) \ln \left(s[\mathrm{M}] / i_{\mathrm{k}}\right)$

from which free nickel concentration can be calculated.

In Figure 4, experimental free nickel concentration is plotted as a function of total nickel added. The theoretical curve was constructed using the speciation program HySS [37] and the experimental conditional stability constants presented in Tables 3 and 4. The good agreement found between experimental and theoretical titration curves shows that free nickel concentration and main species in solution were correctly evaluated.

Since seasonal variations of $\mathrm{pH}$ can be found in xylem sap within the range 4.5-6.5, with lower values in spring and summer and higher in autumn and winter [3], a simulation of chemical speciation of nickel in that $\mathrm{pH}$ range has been done with HySS program. Ni speciation in the model solution of $Q$. ilex xylem sap is dominated by chelation with the carboxylic acids, being the mixed complex of $\mathrm{Ni}$ with oxalic and citric acids the dominant species at $\mathrm{pH}$ 5.5. This is also confirmed in figure 4 where the theoretical curve con-

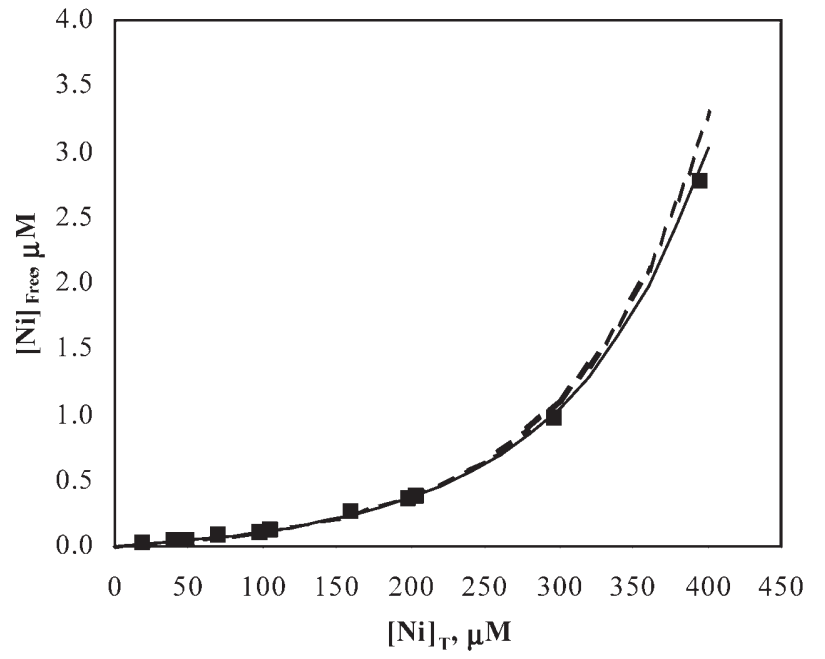

Fig. 4. Titration of the model solution of $Q$. ilex xylem sap with $[$ Asp $]=6.5 \times 10^{-5} \mathrm{M},[\mathrm{His}]=2.0 \times 10^{-5} \mathrm{M},[\mathrm{Ox}]=6.1 \times 10^{-4} \mathrm{M}$, and $[\mathrm{Cit}]=5.0 \times 10^{-4} \mathrm{M}$ with nickel. Experimental points ( $\left.\mathbf{(}\right)$. Theoretical curves assuming: formation of monocomplexes (His, Asp, Cit, Oxal), dicomplexes (Oxal, Cit) and three mixed complexes (full line); formation of mono, di and mixed complexes of citric and oxalic acids (- - -). Voltammetric conditions: $E_{\mathrm{i}}=$ $-800 \mathrm{mV} ; \quad E_{\mathrm{f}} \quad=-1300 \mathrm{mV} ; \quad E_{\mathrm{SW}}=25 \mathrm{mV} ; \quad \Delta E=5 \mathrm{mV}$; frequency $=100 \mathrm{~Hz} ; \quad T=22 \pm 1^{\circ} ; \quad$ electrolyte: $0.10 \mathrm{M} \quad \mathrm{KNO}_{3}$; $\mathrm{pH} 5.5$. 
structed taking into account just the complexes with the carboxylic acids is shown.

\subsection{Titration of a Solution of Xylem Sap from Q. ilex with $\mathrm{Ni}$}

To validate the proposed model the complexation of $\mathrm{Ni}$ by the xylem sap was also studied. Xylem sap samples of $Q$. ilex growing in pots were used. The concentrations for both carboxylic acids and amino acids, with exception of histidine, were within the range of values found in xylem sap of trees growing on serpentine soils (Table 1): citric acid, $1.0 \times$ $10^{-3} \mathrm{M}$; oxalic acid, $1.6 \times 10^{-3} \mathrm{M}$; aspartic acid $9.7 \times 10^{-5} \mathrm{M}$; histidine, $4.7 \times 10^{-7} \mathrm{M}$.

$\mathrm{AC}$ experiments revealed the existence of xylem sap components adsorption on the electrode. However, this could be overcome diluting xylem sap samples in $0.10 \mathrm{M}$ $\mathrm{KNO}_{3}$. The voltammetric behavior of $\mathrm{Ni}(\mathrm{II})$ was then analyzed in the diluted xylem sap solutions as previously described. Again Ni(II) reduction showed to be an irreversible process not affected by adsorption. The xylem sap solutions were then titrated with $\mathrm{Ni}$. As observed during the course of the model solution titration, increasing of total nickel concentration results in a peak current increase being the values however, lower than the corresponding ones for the calibration plot. As to peak potentials of SW voltammograms a cathodic shift was again observed i.e., Ni reduction from the xylem sap solution occurs at a more negative potential than $\mathrm{Ni}$ reduction in the noncomplexing medium. With the increase of nickel in solution, peak potentials tend to shift anodically. Free nickel concentrations were then calculated using Equation 14.

In Figure 5, experimental free nickel concentration is plotted as a function of total nickel added to a xylem sap sample diluted 1:10 with supporting electrolyte. The theoretical curve was constructed taking into account the complexes with citric and oxalic acids. The good agreement found between experimental and theoretical titration curves shows that free nickel in xylem sap was correctly determined and that the main complexes present in xylem sap are those formed with the carboxylic acids.

\section{Conclusions}

Square-wave voltammetry was successfully used to analyze nickel complexation both in model and real solutions of xylem sap of $Q$. ilex.

1. In order to understand the reactive properties of complex systems such as xylem sap, knowledge on the kinetics of the mechanisms involved is mandatory. In a time scale comparable to the one used in SWV (of the order of milliseconds), Ni complexes with oxalic and citric acids behave as semidynamic as well as the mixed complexes with these ligands, while those with amino acids are inert.

2. As to Ni complexation in the model solution of xylem sap, formation of both simple and mixed complexes

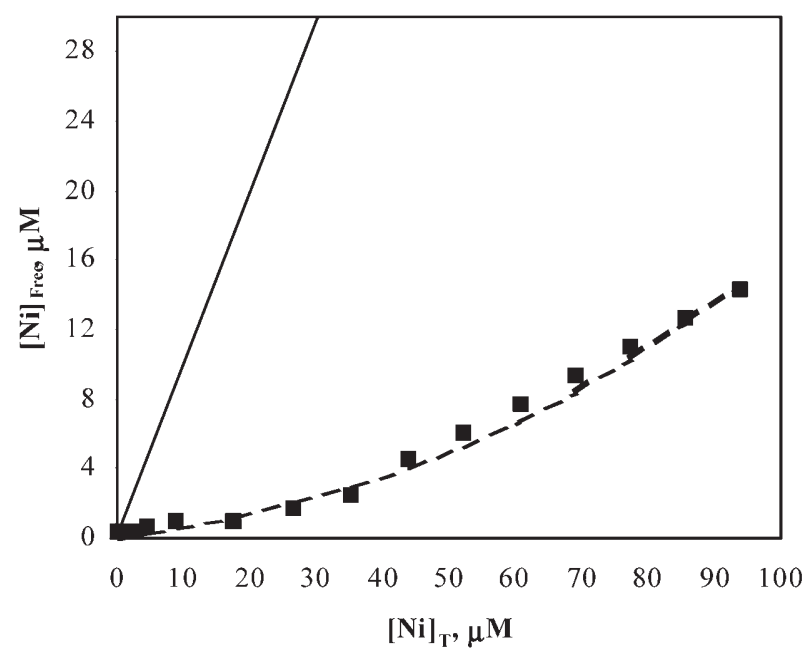

Fig. 5. Titration of $Q$. ilex xylem sap diluted solution with nickel. Experimental points ( $\mathbf{m})$. Theoretical curve assuming formation of mono, di and mixed complex of oxalic and citric acids. The straight line corresponds to calibration (absence of ligands). Voltammetric conditions: $E_{\mathrm{i}}=-800 \mathrm{mV} ; E_{\mathrm{f}}=-1300 \mathrm{mV} ; E_{\mathrm{SW}}=$ $25 \mathrm{mV} ; \Delta E=5 \mathrm{mV}$; frequency $=10 \mathrm{~Hz} ; T=22 \pm 1^{\circ} \mathrm{C}$; electrolyte: $0.10 \mathrm{M} \mathrm{KNO}_{3} ; \mathrm{pH} 5.5$.

occurred. The good agreement between experimental and theoretical titration curves shows that free $\mathrm{Ni}(\mathrm{II})$ concentration and the main species in solution were correctly evaluated. Complexes with carboxylic acids dominate $\mathrm{Ni}$ speciation, being a mixed complex the prevailing species at xylem sap $\mathrm{pH}$. This is important to emphasize since the significance of mixed complexes in mixed ligand environments is often neglected. In fact, they can be even dominant species, although frequently at a very well delimited concentrations range of metal ions and ligands [38].

3 . As to Ni complexation in xylem sap of $Q$. ilex, the good agreement between experimental and theoretical titration curves shows that the main species were correctly evaluated. $\mathrm{Ni}(\mathrm{II})$ speciation is dominated by the complexes formed with carboxylic acids that behave as semidynamic on a time scale of the order of milliseconds. Free Ni(II) ion in xylem sap solution was correctly estimated from the voltammetric data using Equation 14. This means that in any xylem sap sample of $Q$. ilex, the determination of free $\mathrm{Ni}(\mathrm{II})$ concentration is possible, if nickel is present above the technique detection limit.

4. Different tolerance mechanisms may be responsible for plant adaptation to high concentrations of heavy metals on the soil that can be summarized in the plant capacity either to limit metal uptake or to accumulate the metal in nontoxic forms. Nickel complexes with amino acids, since they are inert, are in principle less toxic species and can be transferred more easily to leaves. However, this effect may not be significant since they exist in a small fraction in xylem sap of Q. ilex. Nickel in xylem sap is mainly in the form of semidynamic species that can be more easily exchanged with other components of the medium, namely the partially esterified polygalacturonic acid, the main component of 
xylem wall pectins, which form less dynamic species with nickel [39]. This possibly explains why Quercus ilex growing on serpentine and nonserpentine soils shows only significant differences of Ni concentrations in wood and bark.

\section{Acknowledgement}

This work is within the research project POCTI/1999/MGS/ 35653. The authors thank FCT for the financial support.

\section{References}

[1] J. Proctor, Tree 1999, 14, 334.

[2] E. M. Sequeira, A. R. Pinto, A. Silva, in The Ecology of Areas with Serpentinized Rocks. A World View (Eds: B. A. Roberts, J. Proctor), Kluwer Academic Publishers, Dordrecht 1991, pp. 169-197.

[3] C. Nabais, Ph.D. Thesis, Universidade de Coimbra, Coimbra 2000.

[4] H. Marschner, Mineral Nutrition of High Plants, Academic Press, London 1995.

[5] C. Nabais, J. Hagemeyer, H. Freitas, J. Plant Physiol. 2004, $1625,603$.

[6] K. J. Wilkinson, J. Buffle, in Physicochemical Kinetics and Transport at Biointerfaces (Eds: H. P. van Leeuwen, W. Koster), Wiley, Chichester 2004, pp. 445-533.

[7] H. P. van Leeuwen, in In Situ Monitoring of Aquatic Systems: Chemical Analysis and Speciation (Eds: J. Buffle, G. Horvai), Wiley, Chichester 2000, pp. 253-278.

[8] A. J. Bard, L. R. Faulkner, Electrochemical Methods, Fundamentals and Applications, Wiley, New York 2001.

[9] H. A. Flaschka, EDTA Titrations. An Introduction to Theory and Pratice, Pergamon Press, London 1959.

[10] E. G. Bollard, Aust. J. Biol. Sci. 1957, 10, 292.

[11] J. Osteryoung, J. J. O'Dea, in Electroanalytical Chemistry (Ed: A. Bard), Vol. 14, Marcel Dekker, New York 1986.

[12] A. M. Mota, M. M. Correia dos Santos, in Metal Speciation and Bioavailability in Aquatic Systems (Eds:D. Turner, A. Tessier), Wiley, Chichester 1995, pp. 205-258.

[13] M. L.S. Gonçalves, M. M.C. dos Santos, Sci. Total Environ. 1988, 76,1 .

[14] M. Lovric, S. Komorsky-Lovric, R. W. Murray, Electrochim. Acta 1988, 33, 739.
[15] J. Urbanska, J. Biernat, J. Electroanal. Chem. 1981,130, 141.

[16] M. Eigen, Ber. Bunsenges. Phys. Chem., 1963, 67, 753.

[17] D. W. Margerum, G. R. Cayley, D. C. Weatherburn, G. K. Pagenkopf, in Coordination Chemistry (Ed: A. Martell), Vol. 2, ACS Monograph 174, ACS, Washington 1978.

[18] F. M. M. Morel, J. G. Hering, Principles and Applications of Aquatic Chemistry, Wiley Interscience, New York 1983.

[19] J. Heyrovsky, J. Kuta, Principles of Polarography, Publishing House of the Czechoslovak Academy of Sciences, Prague 1965.

[20] A. E. Martell, R. M. Smith, Critical Stability Constants, Plenum Press, New York 1976.

[21] Metal Ions in Biological Systems, Vol. 2 (Ed: H. Siegel), Marcel Dekker, New York 1974.

[22] F. A. Cotton, G. Wilkinson, C. A. Murillo, M. Bochmann, Advanced Inorganic Chemistry, Wiley, Chichester 1999.

[23] V. S. Sharma, J. Schubert, J. Chem. Ed. 1969, 46, 506.

[24] N. E. Jackobs, D. W. Margerum, Inorg.Chem. 1967, 11, 2038.

[25] D. D. Perrin, V. S. Sharma, J. Chem. Soc. (A) 1968, 2, 446.

[26] P. G. Daniele, G. Ostacoli, J. Inorg. Nucl. Chem. 1978, 40, 1273.

[27] M. R. Ullah, P. Bhattacharya, Bull. Chem. Soc. Jpn. 1991, 64, 3659.

[28] J. Maslowska, L. Chruscinski, Polyhedron 1984, 3, 523.

[29] E. Farkas, E. A. Enyedy, G. Micera, E. Garribba, Polyhedron 2000, 19, 1727

[30] R. N. Patel, N. Singh, R. P. Shrivastava, K. K. Shukla, P. K. Singh, Proc. Indian Acad. Sci. (Chem. Sci.) 2002, 114, 115.

[31] G. Ruiz-Cabrera, J. C. Rodríguez-Placeres, F. Ramos-Steffens, A. Arévalo, Polyhedron 1985, 4, 221.

[32] H. M. Killa, E. S. M. Mabrouk, M. M. Ghoneim, Collect. Czech. Chem. Commun. 1991, 56, 1193.

[33] M. I. Ismail, Talanta 1992, 39, 1107.

[34] C. Rodríguez-Placeres, G. Ruiz-Cabrera, A. Alloza, A. Arévalo, Collect. Czech. Chem. Commun. 1988, 53, 506.

[35] M. Zelic, Anal. Chim. Acta 1993, 271, 275.

[36] F. Berbel, J. M. Díaz-Cruz, C. Ariño, M. Esteban, J. Electroanal. Chem. 1997, 431, 99.

[37] L. Alderighi, P. Gans, A. Ienco, D. Peters, A. Sabatini and A. Vacca, Coord. Chem. Rev. 1999, 184, 311. Programme HySS may be downloaded, free of charge, at http://www.hyperquad.co.uk/index.htm

[38] W. Stumm, J. Morgan, Aquatic Chemistry, Wiley, Chichester 1998.

[39] C. Vilhena, A. M. Mota, M. L. S. Gonçalves, submitted for publication. 